\title{
Atmospheric stability and diurnal patterns of aeolian saltation on the Llano Estacado
}

(Received ?; revised ?; accepted ?. - To be entered by editorial office)

Aeolian transport is driven by aerodynamic surface stress imposed by turbulent winds in the Earth's atmospheric boundary layer (ABL). ABL regime is influenced by stratification, which can either enhance or suppress production of turbulence by shear associated with the vertical gradient of streamwise velocity. During the day and night, surface heat fluxes induce a negative (unstable) and positive (stable) vertical gradient of potential temperature, respectively, which modifies the role of buoyancy in turbulence production. During the brief morning and evening transition periods, the vertical gradient of potential temperature vanishes (neutral stratification). The Monin-Obukhov similarity theory describes how the vertical gradient of streamwise velocity varies with stratification. Simultaneous field measurement of wind speed and aeolian activity were obtained over a 218-day period on a bare, sandy surface on the high plains of the Llano Estacado region of west Texas. Wind speed was measured at a height of $2 \mathrm{~m}$ with a propeller-type anemometer and aeolian activity was measured at the surface with a piezoelectric saltation sensor. We have used the wind speed measurements within the framework of the Monin-Obukhov similarity theory to estimate "typical" shear velocity, $u_{*}$, of the ABL as stratification is varied (characterized with the stability parameter). This approach results in a color flood contour of $u_{*}$ against time of day and stability parameter: the procedure demonstrates that aeolian activity is most likely to occur during the day, when buoyancy acts in conjunction with mechanical shear to increase $u_{*}$.

Key words: Authors should not enter keywords on the manuscript, as these must be chosen by the author during the online submission process and will then be added during the typesetting process (see http://journals.cambridge.org/data/relatedlink/jfmkeywords.pdf for the full list)

\section{Introduction and background}

During periods of aeolian activity, turbulent winds exert aerodynamic forces on the surface that destabilize stationary sediment grains such that they become detached and move downwind. As grains move under the combined forces of wind and gravity they saltate or skip across the surface. Upon downwind impact, sediment grains perpetuate aeolian saltation by "splashing" other grains from the sediment bed (Shao 2008; Kok et al. 2012; Pahtz et al. 2012). Predictions of aeolian saltation mass transport, $Q$, are typically based upon shear velocity, $u_{*}$, associated with the atmospheric boundary layer (ABL) raised to the third power. For example, consider the Bagnold scheme (Bagnold 1937, 1956; Martin et al. 2013; Kok et al. 2012):

$$
Q=C_{B}\left(\frac{D_{p}}{D_{250}}\right)^{1 / 2} \frac{\rho_{a}}{g} u_{*}^{3}
$$


where $C_{B} \sim \mathcal{O}\left(10^{0}\right)$ is a dimensionless empirical parameter, $\rho_{a}$ is the density of air, $D_{p}$ is the aggregate particle diameter, $D_{250}=250 \mu \mathrm{m}$ is a reference particle diameter, and $g$ is gravity (the efficacy of Eq. 1.1-style relations is itself an open question, Martin et al. 2013; Kok et al. 2012). One may also consider classic relations for $u_{*, t}$, the ABL shear velocity at which sediment is mobilized; consider, for example, the Bagnold scheme (Bagnold 1956; Shao 2008; Kok et al. 2012):

$$
u_{*, t}=A_{B}\left(\frac{\rho_{p}-\rho_{a}}{\rho_{a}} g D_{p}\right)^{1 / 2},
$$

where $A_{B}=A_{B}\left(R e_{*, t}\right) \sim \mathcal{O}\left(10^{-1}\right)$ is a non-dimensional coefficient related to the particle friction threshold velocity, $R e_{*, t}=u_{*, t} D_{p} / \nu$, and $\rho_{p}$ is sand grain density. These equations demonstrate how turbulence, as $u_{*}={\overline{u^{\prime} w^{1}}}^{1 / 2}$ (where $u$ and $w$ correspond with the prevailing streamwise and surface-normal flow directions, respectively, and "prime" denotes fluctuation from time average), influences aeolian activity. It follows that the prevailing conditions responsible for setting the ABL $u_{*}$ are critically important to aeolian activity. We note also that diurnal variability of thermal conditions, the topic of this work, would affect changes in $\rho_{a}$ that would in turn alter $Q$ and $u_{*, t}$ (Eqs. 1.1 and 1.2, respectively). However, it should be stressed that since $Q \sim \rho_{a}$ and $u_{*, t} \sim \rho_{a}^{-1 / 2}$, such $\rho_{a}$ changes will not substantially alter the results.

ABL regime is categorized based on stratification, or vertical gradient of potential temperature, $\partial \theta(z) / \partial z$, which is itself set by solar forcing (Stull 1988) (here, $z$ represents the coordinate orthogonal to Earth's surface). The unstable, neutral, and stable stratification regimes correspond with $\partial \theta(z) / \partial z<0,=0$, and $>0$, respectively. Solar forcing during daylight hours results in $\partial \theta(z) / \partial z<0$, which drives convective mixing and production of turbulence via buoyant, turbulent plumes (that is, turbulence is produced by buoyancy and mechanical shear associated with $\partial U(z) / \partial z>0$, where $U(z)$ is the mean streamwise velocity profile). At night, when $\partial \theta(z) / \partial z>0$, turbulence produced by shear is attenuated by buoyancy (Mahrt 1998). The brief morning- and evening-transitions correspond with $\partial \theta(z) / \partial z=0$, during which the ABL behaves as a canonical turbulent half channel (Deardorff 1972; Csanady 1974; Mason 1994; Wyngaard 2010). The buoyancy forcing, $f_{B}$, associated with potential temperature gradients are incorporated via the Boussinesq approximation (Cushman-Roisin \& Beckers 2011; Wyngaard 1979) in the vertical momentum transport equation, $f_{B}=g \alpha \delta T(\boldsymbol{x}, t)=g\left(1-\rho(\boldsymbol{x}, t) / \rho_{0}\right)$, where $\alpha$ is thermal conductivity. With this, we see how $f_{B}$ alters vertical momentum fluctuations, $w^{\prime}$, and therefore the magnitude of $u_{*}$.

Since convective mixing enhances $u_{*}$, and sediment mass flux (Eq. 1.1) scales as $Q \sim u_{*}^{3}$, one may deduce that - in general - aeolian activity is greatest during the day. Indeed, this has been reported based on anecdotal observations and detailed field measurement (Stout 2007, 2010; Frank \& Kocurek 1994). In this document, we first report on a field campaign that was conducted on a sandy landscape on the Llano Estacado region of West Texas (see Fig. 1 for map showing testing site). This field campaign provided a 218 day timeseries (sampled at $1 \mathrm{~Hz}$ ) of wind speed and aeolian activity, and clearly demonstrated that aeolian processes are most active during the day and most pronounced in the early afternoon. We then frame these observations within the Monin-Obukhov similarity theory (MOST) (Monin \& Obukhov 1954), which provides an analytical means to demonstrate how atmospheric stability (as a prognostic indication for time of day) affects the ABL's $u_{*}$. We use $u_{*, t}$ determined based on field data from the site (we could have, alternatively, used the Eq. 1.2 Bagnold scheme to compute $u_{*, t}$ for the landscape; Bagnold 1956; Shao 2008), and demonstrate that $u_{*} / u_{*, t}>1$ occurs for unstable stratification; that is, under 


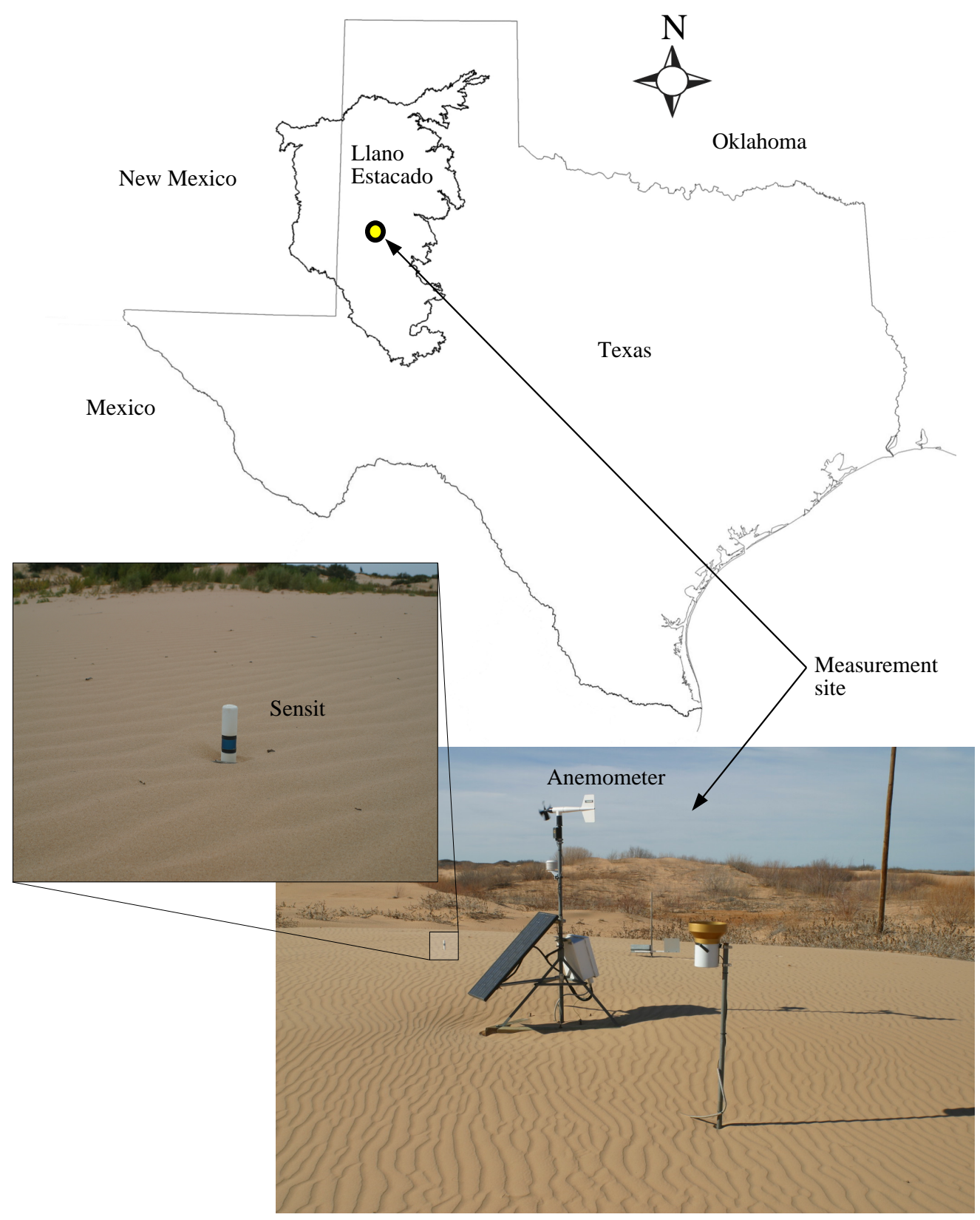

Figure 1: (Color) Imagery of field measurement system. Top map shows regional map, political boundaries, outline of Llano Estacado region in west Texas and eastern New Mexico, and indication of measurement site. Photographs show in situ field sampling system for simultaneous measurement of wind speed (propeller-style anemometer) and aeolian saltation activity (Sensit piezoelectric aeolian saltation sensor). 
"typical" geostrophic conditions, the ABL is only able to mobilize sediment during the day. Furthermore, we use the momentum balance equations - reduced for conditions resembling the ABL - to show how prevailing geostrophic winds affect $u_{*}$ and could mobilize sediment in the absence of convective heating (or could combine to enable maximum $Q$ ). Sec. 2 presents details of the field campaign and resulting datasets. In Sec. 3 , we show results incorporating the field datasets with the similarity theory to attain estimates of aeolian activity under varying ABL stratification and prevailing geostrophic conditions. Concluding remarks are offered in Sec. 4.

\section{Field campaign}

A portable sampling system (Fig. 1) was designed and constructed for this field study. The primary system components included a small meteorological tower, a piezoelectric saltation sensor and a datalogger. The system was powered by a 12 -volt battery, which was charged during the day by a solar panel.

Sand movement was detected with a commercially available saltation sensor called Sensit (Fig. 1). The Sensit produces a pulse signal each time a saltating grain impacts its piezoelectric transducer. A continuous record of saltation activity was obtained by sampling the output from the Sensit at a frequency of $1 \mathrm{~Hz}$. During periods of active sand transport, the piezoelectric transducer produced a signal that was used as an on- or off-indicator of aeolian sediment transport. If one or more impacts were detected during a given second then that second was registered as one "saltation second". Saltation seconds were summed at the end of each five-minute period to obtain the total number of seconds that blowing sand was detected and then the counter was reset to zero, and the process was repeated during the next five-minute period (Stout 2007).

Instruments mounted on the meteorological tower measured climatic variables such as wind speed and direction, air temperature, relative humidity and rainfall. Wind velocity was measured at a height of $2 \mathrm{~m}$ with a combined anemometer and wind direction indicator (Fig. 1). Other meteorological sensors were mounted at a height of $1 \mathrm{~m}$ above the sand surface. These included a tipping-bucket rain gauge with a resolution of $0.1 \mathrm{~mm}$ per tip and a combined temperature and relative humidity sensor. All meteorological variables were sampled at a frequency of $1 \mathrm{~Hz}$ and averaged every five minutes.

The site selected for this study was a level surface of fine sand located at the eastern edge of an extensive sand sheet that stretches across the Llano Estacado from the Pecos River Valley through Lea County, New Mexico, and across portions of Yoakum, Cochran, and Terry counties of western Texas (Holliday 2001). The location of the measurement site within the Llano Estacado region is shown in Fig. 1. The sampling system was installed near the center of a large, nearly circular, sandy surface $300 \mathrm{~m}$ in diameter. The highly mobile sands at the sampling site contrasted sharply with the surrounding low-relief dunes that are mostly stabilized by vegetation (Stout 2007).

Sand samples were obtained and sieved to obtain the particle size distribution. The analysis revealed that these sands are properly classified as "fine sand" with a median grain diameter of $180 \mu \mathrm{m}$. Less than $10 \%$ of the grains were found to be smaller than $110 \mu \mathrm{m}$ and only $8 \%$ had diameters greater than $250 \mu \mathrm{m}$. Using Eq. 1.2 with $D_{p}=$ $180 \mu \mathrm{m}, \rho_{p}=2650 \mathrm{~kg} / \mathrm{m}^{3}$ (Stout \& Zobeck 1996), $\rho_{a}=1.2754 \mathrm{~kg} / \mathrm{m}^{3}$, and $A_{B}=0.1$ (see (Shao 2008), Fig. 5.12c), we estimate that $u_{*, t}=0.19 \mathrm{~m} / \mathrm{s}$ for the sandy test site. This is less than the value derived from post-processing of measured data, which indicated $u_{*, t}=0.36 \mathrm{~m} / \mathrm{s}$ (Stout 2007); we have adopted $u_{*, t}=0.36 \mathrm{~m} / \mathrm{s}$ for this report (although the conclusions drawn from this study are invariant to $u_{*, t}$ ). Moreover, the arguments presented here are contingent upon a single $u_{*, t}$, when in reality there could be a range 
132

of values owing to distribution of sediment sizes at the site. And since $u_{*, t} \sim D_{p}^{1 / 2}$, we expect $u_{*, t}$ to be relatively insensitive to $D_{p}$.

Sampling began on 25 September 2002 and extended through 1 May 2003, a period of roughly seven months. On the high plains of the Llano Estacado, late fall, winter and early spring seasons are typically windy and relatively dry compared to the rest of the year. Due to the highly mobile nature of the sand surface, the site was visited frequently to ensure that drifting sand did not cover the Sensit. Often, the height of the Sensit had to be adjusted so that the piezoelectric sensor remained within a target range of between 0 and $5 \mathrm{~cm}$ (Fig. 1 inset shows the Sensit instrument). At the test site, we estimate that the aerodynamic roughness length is $z_{0}=0.005 \mathrm{~m}$ (Brutsaert 2005).

The resulting dataset was used to generate an ensemble mean diurnal variation of streamwise velocity, $U(z, t)$, at elevation $z=2 \mathrm{~m}$ (Stout 2007, 2010) and simultaneous measurement of aeolian saltation activity to formulate an aeolian saltation indicator. These datasets have comprehensively shown that sediment mobilization is greatest in the early afternoon (see, for example, Fig. 5 of Stout 2010). During the atmosphere's diurnal cycle, surface heating induces buoyant turbulent plumes that produce turbulence (in conjunction with production by mechanical shear). Below, we incorporate the field datasets within the Monin-Obukhov similarity theory to demonstrate that production of turbulence via mechanical shear and convective mixing is responsible for observations that aeolian activity is greatest during the day hours.

\section{Monin-Obukhov similarity theory and momentum balance}

\subsection{Monin-Obukhov similarity theory}

The Monin-Obukhov similarity theory is a framework to define the vertical gradient of mean streamwise velocity in terms of characteristics of the ABL (Garratt 1994; Shao 2008) and a similarity function:

$$
\frac{\partial U(z, \zeta)}{\partial z}=\frac{u_{*}(\zeta)}{\kappa z} \phi_{M}(\zeta)
$$

where $\phi_{M}(\zeta)$ is the (momentum) similarity function, $\kappa=0.4$ is the von Kármán constant, and $\zeta=z / L$ is the stability parameter where $L$ is the Obukhov length, or the elevation at which production of turbulence by buoyancy and mechanical shear is equal:

$$
L=-\frac{\bar{\theta} u_{*}^{3}}{\left.\kappa g \overline{w^{\prime} \theta^{\prime}}\right|_{0}}
$$

where $\bar{\theta}$ is the mean surface temperature, and $\left.\overline{w^{\prime} \theta^{\prime}}\right|_{0}$ is the surface heat flux. $\phi_{M}(\zeta)$ can be modeled as:

$$
\phi_{M}(\zeta)=\left\{\begin{aligned}
\left(1-\gamma_{M} \zeta\right)^{-1 / 4} & \text { for } \zeta \leqslant 0 \text { (unstable), or } \\
1+\beta_{M} \zeta & \text { for } \zeta>0 \text { (stable). }
\end{aligned}\right.
$$

where $\gamma_{M} \approx 16$ and $\beta_{M} \approx 5$ are empirical model parameters (Garratt 1994) for the similarity function, $\phi_{M}(\zeta)$. More recent $\phi_{M}(\zeta)$ models could have been used, although none would have substantively influenced conclusions reached in the present study; for example, see Horst et al. (2004) or Kumar et al. (2006). Integration of Eq. 3.1 yields the mean streamwise velocity profile,

$$
\frac{U(z, \zeta)}{u_{*}}=\frac{1}{\kappa} \psi_{M}(z, \zeta),
$$


where $\psi_{M}(z, \zeta)=\int_{z_{0} / L}^{z / L} \frac{1}{\zeta}(1-\phi(\zeta)) d \zeta$ is the stability correction parameter which, upon integration, yields:

$$
\psi_{M}(z, \zeta)= \begin{cases}\ln \left(\frac{z}{z_{0}}\right)-\psi_{1}(\zeta) & \text { for } \zeta \leqslant 0 \text { (unstable), or } \\ \ln \left(\frac{z}{z_{0}}\right)+\beta_{M} \zeta & \text { for } \zeta>0 \text { (stable), }\end{cases}
$$

where $\psi_{1}=2 \ln \left(\frac{1+\phi_{M}(\zeta)^{-1}}{2}\right)+\ln \left(\frac{1+\phi_{M}(\zeta)^{-2}}{2}\right)-2 \tan ^{-1}\left(\phi_{M}(\zeta)^{-1}\right)+\frac{\pi}{2}$. Fig. 2 shows $\psi_{M}(z, \zeta)$ for $-2 \leqslant \zeta \leqslant 2$ (this $\zeta$ range being consistent with ambient measured values; Kleissl et al. 2004a; Horst et al. 2004; Kumar et al. 2006). From Eqs. 3.3 and 3.5, the friction velocity can evaluated:

$$
u_{*}(\zeta)=\frac{\kappa U(z)}{\psi_{M}(z, \zeta)} .
$$

MOST is a concept strictly valid for the atmospheric surface layer, which occupies the lowest $\approx 10 \%$ of the ABL (Monin \& Obukhov 1954; Businger et al. 1971). The lowest region of the surface layer is occupied by the so-called roughness sublayer (Raupach et al. 1991; Garratt 1994; Brutsaert 2005), which is affected by individual topographic elements and landscape heterogeneities. In order to demonstrate efficacy of the present study, we provide some rudimentary scaling arguments to demonstrate that the singlepoint measurements were indeed retrieved from the surface layer and thus can be used within MOST to infer $u_{*} . z_{0}=0.005 \mathrm{~m}$ for the measurement site (see Section 2; Brutsaert 2005; Stout 2007, 2010), from which we can infer aggregate obstacle elevation, $h$, using the model of Garratt (1994):

$$
\frac{z_{0}}{h}=\gamma_{1}\left(1-\frac{d}{h}\right)
$$

where $d$ is the zero-plane displacement, $h$ is the canopy or element height, and $0.2 \lesssim$ $\gamma_{1} \lesssim 0.4$. We can assume that $d / h=0.5$ and $\gamma_{1}=0.2$, indicating that $z_{0} / h \approx 0.1$ or $h \approx 0.05 \mathrm{~m}$ (representing intermittent patches of low vegetation and sand ripples characteristic of the site); further, we emphasize that $z_{\text {Inst. }} / h=40$, where $z_{\text {Inst. }}=2 \mathrm{~m}$ is the instrument height (Section 2). We also note that the roughness sublayer depth, $z_{\mathrm{RSL}}$, is typically $3 \lesssim z_{\mathrm{RSL}} / h \lesssim 5$ (Raupach et al. 1991). At the site, we conclude that $z_{\mathrm{RSL}} \approx 0.2 \mathrm{~m}$, or $z_{\text {Inst. }} / z_{\mathrm{RSL}} \approx 10$. This exercise demonstrates that the sampling elevation is above the roughness sublayer but within the surface layer. Moreover, recall from Sec. 2 that the measurements were made at the approximate geometric center of a $300 \mathrm{~m}$ diameter sandy surface, surrounded by small dunes and weakly undulating terrain (as is typical of landscapes on the Llano Estacado; Stout 2007). Thus, any "edge effects" or "flow development" will have been thoroughly completed before winds encounter the measurement site, ensuring that the ABL exhibits horizontally statistically homogeneity at the site. In practice, a vertically-staggered series of measurements would be preferable. In the absence of this, however, the very large number of diurnal ensembles and clear surface layer elevation measurements render the present measurements suitable (including the 5-minute averages).

With this, we can substitute the $U(z, t)$ time-series recorded via field campaign (see Sec. 2) and $-2 \leqslant \zeta \leqslant 2$ into Eq. 3.6 to attain the Fig. 3(a) color flood contour. The figure includes annotations for the neutral (channel) case (vertical orange line) and the approximate morning- and evening-transitions. Moreover, we have superimposed $u_{*, t}=$ $0.36 \mathrm{~m} / \mathrm{s}$ for the site (as determined by post-processing of field measurement data; Stout 


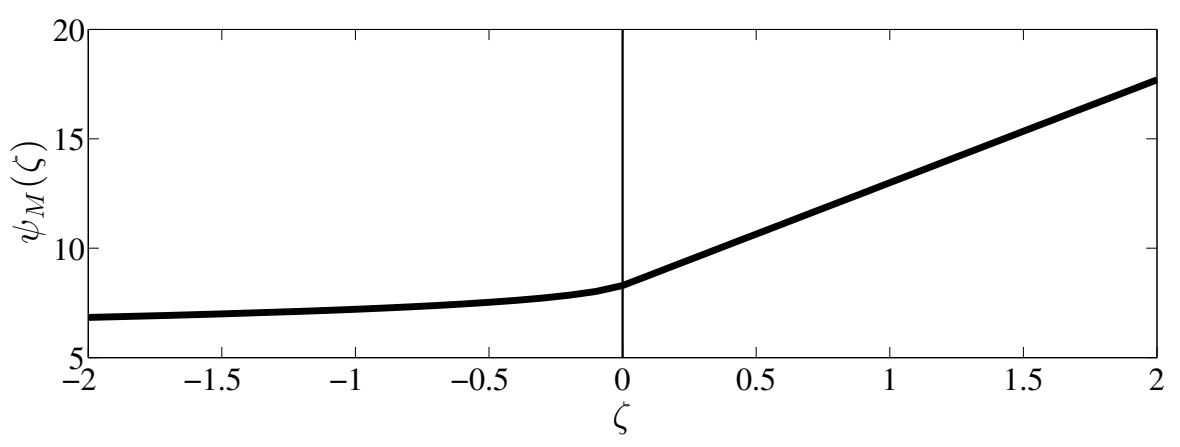

Figure 2: Stability correction parameter (Eq. 3.5) for range of stability parameters.

2007 ) on the figure. By including $u_{*, t}$, we can infer the ambient conditions under which sediment is mobilized by aeolian saltation. As reported by Stout (2010), aeolian activity is most pronounced during the day at the site. Fig. 3(a) demonstrates that the ABL $u_{*}$ exhibits a strong diurnal evolution. During the day, when the ABL stratification is unstable and $\zeta<0$, we see a broad time interval over which sediment could be mobilized (i.e., $-2 \leqslant \zeta \leqslant 0.5$ and 09:00 $\lesssim t(\mathrm{hr}) \lesssim 18: 00)$. Similarly, the figure suggests that during the night (i.e., 00:00 $\lesssim t(\mathrm{hr}) \lesssim 06: 00$ and 18:00 $\lesssim t(\mathrm{hr}) \lesssim 24: 00)$, when $\zeta>0$, the ABL would not develop the needed $u_{*}$ to mobilize sediment. We emphasize that the Fig. 3(a) contour is predicated on the ensemble-averaged diurnally-varying velocity timeseries, with 218 days (ensembles). Given the number of ensembles, intermittent periods of elevated shear $\left(u_{*}\right)$ associated with regional/mesoscale circulations (occurring, for example, at night or during dust storm periods; Lee et al. 2012) are "smeared" from the Fig. 3(a) result. For reference, Fig. 3(b) is a vertical profile of the so-called "saltation activity" aeolian saltation indicator function:

$$
\gamma(t ; \delta t)=R(i(t)) \frac{\delta t}{T},
$$

where $R(x)$ is the ramp function, defined as $R(x)=1$ if $x>0$ and $R(x)=0$ otherwise, and $i(t)$ is the sum of number of impacts by sediment grains on the Sensit piezoelectric sensor (see also Fig. 1 and Sec. 2), $\delta t$ is the time increment for characterizing saltation activity, and $T$ is the time increment between sampling; for the Stout (2010) dataset, $\delta t$ and $T$ are 1 and 300 seconds, respectively. The $\gamma(t ; \delta t)$ profile has been re-scaled in order to fit within the horizontal figure limits; however, since $\gamma(t ; \delta t)$ is strictly an indicator function, it can be re-scaled with no loss of generality. The profile clearly demonstrates that aeolian saltation rises mid-morning, asymptotes during the mid-afternoon, and reverts to a "minimal state" at around 19:00. The $\gamma(t ; \delta t)$ profile is similar to the locus of $u_{*, t}$ points corresponding with $u_{*}=u_{*, t}$ on Fig. $3(\mathrm{a})$. This further demonstrates that, statistically, it is during the day - when $\zeta<0$ - that the ABL is more likely to develop a $u_{*}$ adequate to mobilize sediment. However, comparison of Figs. 3(a) and 3(b) also shows that unstable stratification alone is not necessary to mobilize sediment; rather, very strong winds can overcome buoyant suppression of turbulence and drive aeolian saltation at night. Next, we use the streamwise and transverse momentum transport equations to demonstrate how variations of the prevailing regional circulations affect $u_{*}$.

\subsection{Geostrophic conditions}

Larger (mesoscale) dynamics invariably change geostrophic wind conditions aloft the test site. Consider the streamwise $(x)$ and transverse $(y)$ momentum balance equations under 

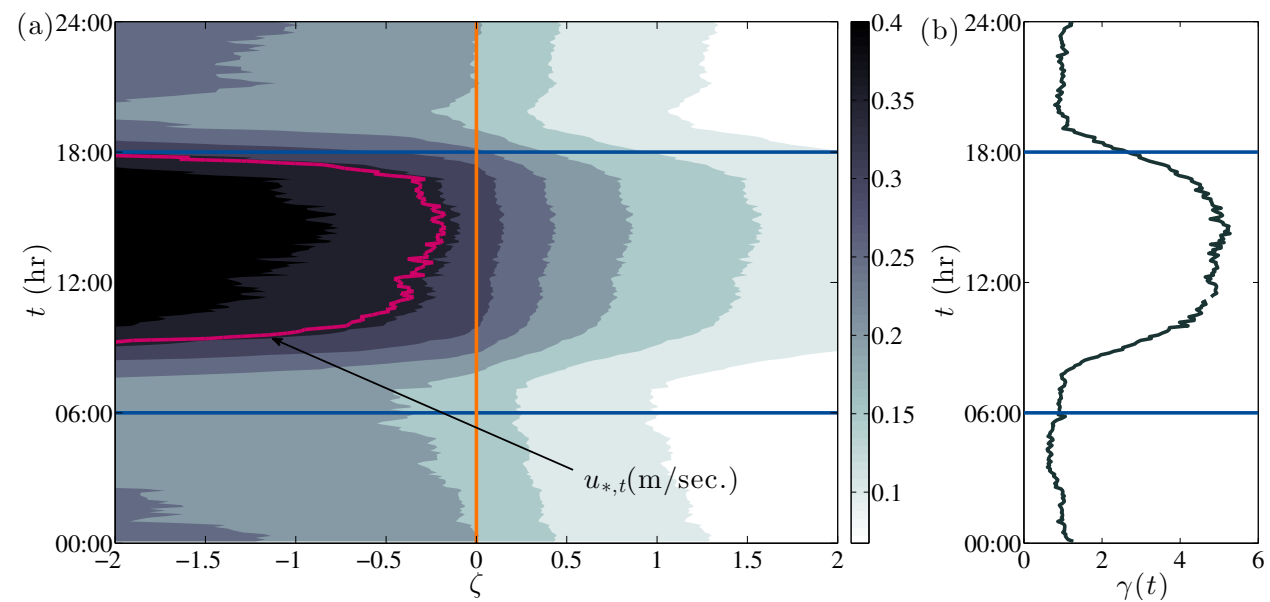

Figure 3: (Color) Aerodynamic characteristics of diurnally-evolving atmospheric boundary layer and aeolian saltation: Panel (a) shows contour of diurnal variability of threshold velocity against stability parameter; for reference, vertical orange line denotes neutral (channel) case and cerise line denotes $u_{*, t}$ as predicted with Eq. 1.2. Panel (b) illustrates vertical profile of saltation activity indicator function, $\gamma(t ; \delta t)$ (Stout 2010). On both panels, horizontal blue lines denoting 06:00 and 18:00 local time are added to highlight morning and evening transition times.

fully-developed, horizontally homogeneous conditions:

$$
-\frac{1}{\rho} \frac{\partial p}{\partial x}+f_{c} \tilde{v}-\frac{\partial \overline{u^{\prime} w^{\prime}}}{\partial z}=0
$$

and

$$
-\frac{1}{\rho} \frac{\partial p}{\partial y}+f_{c} \tilde{u}-\frac{\partial \overline{v^{\prime} w^{\prime}}}{\partial z}=0
$$

where $f_{c}=2 \Omega \sin \phi$ is the Coriolis frequency, $\nabla p=(\partial p / \partial x) \hat{i}+(\partial p / \partial y) \hat{j}$ is the pressure gradient, and $\overline{u^{\prime} w^{\prime}}$ and $\overline{v^{\prime} w^{\prime}}$ are (shearing) components of the Reynolds stress tensor, $\boldsymbol{T}=\overline{\boldsymbol{u}^{\prime} \otimes \boldsymbol{u}^{\prime}}$. In the surface layer, wherein the Rossby number (which may be composed as $\left.R o(z, t)=U(z, t) / f_{c} z\right)$ is large, Eqs. 3.9 and 3.10 reduce to a balance between the imposed pressure gradient and divergence of turbulent stresses (Wyngaard 2010). Likewise, in the outer layer where turbulent stresses vanish, Eqs. 3.9 and 3.10 enable introduction of the geostrophic winds, $\boldsymbol{G}=\left\{U_{G}, V_{G}\right\}$, thereby enabling Eqs. 3.9 and 3.10 to be expressed in a slightly different form:

$$
f_{c}\left(\tilde{v}-V_{G}\right)-\frac{\partial \overline{u^{\prime} w^{\prime}}}{\partial z}=0
$$

and

$$
f_{c}\left(U_{G}-\tilde{u}\right)-\frac{\partial \overline{v^{\prime} w^{\prime}}}{\partial z}=0 .
$$

Further reduction leads to an inner- (or surface layer) and outer-layer expression for the atmospheric boundary layer profile (Tennekes \& Lumley 1972). The inner-layer profile has been already shown in Eq. 3.4, while the outer-layer profile is:

$$
\frac{U(z)-U_{G}}{u_{*}}=\frac{1}{\kappa} \ln \left(\frac{z f_{c}}{u_{*}}\right)+C .
$$


Eqs. 3.4 and 3.13 can be equated based on $U(z)$ to attain the relation:

$$
\frac{U_{G}}{u_{*}}=\left\{\begin{array}{cc}
\frac{1}{\kappa}\left(\ln \left[\frac{u_{*}}{z_{0} f_{c}}\right]-\psi_{1}(\zeta)\right)-C & \text { for } \zeta<0 \text { (unstable), } \\
\frac{1}{\kappa}\left(\ln \left[\frac{u_{*}}{z_{0} f_{c}}\right]\right)-C & \text { for } \zeta=0 \text { (neutral), } \\
\frac{1}{\kappa}\left(\ln \left[\frac{u_{*}}{z_{0} f_{c}}\right]+\beta_{M} \zeta\right)-C & \text { for } \zeta>0 \text { (stable). }
\end{array}\right.
$$

We have numerically solved the Eq. 3.14 transcendental relation for $u_{*}$ in response to variations in geostrophic wind and stability parameter, $2 \leqslant U_{G} \leqslant 12$ and $-2 \leqslant \zeta \leqslant 2$ (as opposed to using approximations, for example as proposed by Frandsen et al. 2006; Calaf et al. 2010). The resulting $u_{*}$ color flood contour is shown on Fig. 4. For illustration, we have included $u_{*, t}$ for the Llano Estacado test site (Stout 2007). The figure demonstrates how the combined affect of surface heating (unstable stratification) and mechanical shear (prevailing geostrophic winds) result in elevated $u_{*}$. Consider, for example, a horizontal virtual line spanning the figure for constant $U_{G}=8 \mathrm{~m} / \mathrm{s}$ The biggest $u_{*}$ occurs for the smallest $\zeta=-2$, and $u_{*}$ declines for increasing (more positive) $\zeta$, indicating how ABL forcing would decrease for some imposed $U_{G}$. Note also that for $U_{G}=10 \mathrm{~m} / \mathrm{s}$ and $\zeta \gtrsim-1$, the ABL would not support aeolian saltation at the site. Moreover, for any constant $\zeta$, increasing $U_{G}$ increases the resulting $\mathrm{ABL} u_{*}$. A final way to conceptualize Fig. 4 is by following the $u_{*, t}$ profile on Fig. 4: for $\zeta=-2$, the ABL can mobilize sediment with $U_{G} \approx 9 \mathrm{~m} / \mathrm{s}$, while for increasing (more positive) $\zeta$ the ABL $U_{G}$ must continue to rise in order to mobilize sediment. With this, we show finally the ensemble-averaged diurnal pattern of $2 \mathrm{~m}$ wind speed, $U(z, t)$ (used also for determination of the Fig. 3a $u_{*}$ ) on Fig. 5. For reference, this figure also includes the profiles of $2 \mathrm{~m}$ threshold velocity, $U_{t}(z ; \zeta)$, with varying stability parameter. We considered three values of stability parameter changing within a "typical" range (Horst et al. 2004; Kleissl et al. 2004b; Kumar et al. 2006): $\zeta=-0.5$ (convective stratification), $\zeta=0$ (neutral stratification), and $\zeta=0.5$ (stable stratification), which can be used to determine threshold velocity via:

$$
U_{t}(z ; \zeta)=\frac{u_{*, t}}{\kappa} \psi_{M}(z ; \zeta),
$$

where $u_{*, t}$ was determined from post-processing of field data (Stout 2007). Fig. 5 also includes profiles of the so-called saltation seconds aeolian saltation indicator function (Eq. 3.8 and accompanying text). Owing to the ensemble averaging performed to attain the Fig. $5 \gamma(t ; \delta t)$ profile, saltation appears to occur continuously throughout the diurnal cycle (indeed, Fig. 4 shows how variable $U_{G}$ can mobilize sediment, while convective heating serves simply to augment this process as evidenced in Fig. 3a). The $U_{t}\left(z ; \zeta=\frac{1}{2}\right)$ and $U_{t}(z ; \zeta=0)$ profiles - which corresponds only with the evening hours and neutral conditions, respectively - demonstrates that the saltation threshold would never be met at the site. Moreover, the unstably stratified profile, $U_{t}\left(z ; \zeta=-\frac{1}{2}\right)$, demonstrates that saltation would occur during the day only (i.e. when $\left.U(z, t)>U_{t}\left(z ; \zeta=-\frac{1}{2}\right)\right)$. This demonstrates how enhanced saltation activity can take place once the threshold is exceeded (that is, favorable aloft conditions in the convective regime result in elevated aeolian activity; Bomar 1983; Stout 2001). We note also that $U(z, t)$ rises vigorously for 08:00 $\lesssim t \lesssim 11: 00$, and then increases slowly before reaching a maxima at $t \approx 01: 00$. In contrast, $\gamma(t ; \delta t)$ continues to increase more rapidly during the 08:00 $\lesssim \lesssim 11: 00$ period, which may indicate that $u_{*}$ associated with $U_{G}$ is met (Fig. 4) but additional 


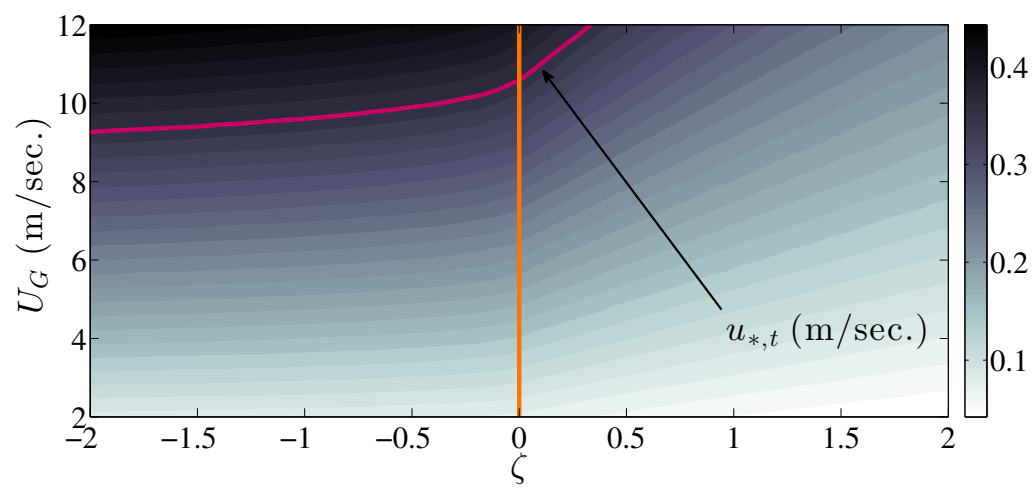

Figure 4: (Color) Color flood contour of $u_{*}$ for $2 \leqslant U_{G} \leqslant 12$ and $-2 \leqslant \zeta \leqslant 2$, as predicted with Eq. 3.14. Vertical orange profile corresponds with $\zeta=0$ (neutral stratification or channel flow).

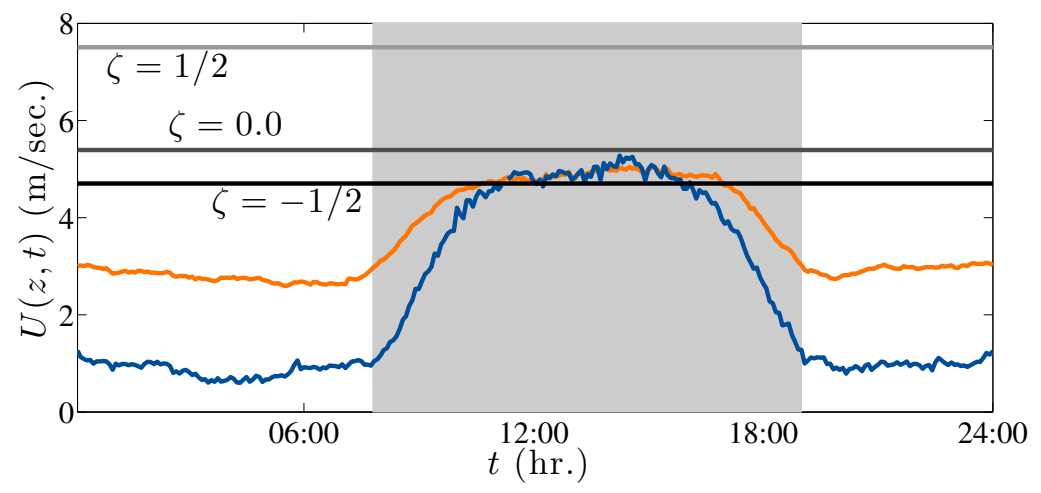

Figure 5: (Color) Diurnal evolution of ensemble quantities from the field campaign: solid orange and blue lines denote $U(z, t)$ and $\gamma(t ; \delta t)$, respectively (note that $\gamma(t ; \delta t)$ has been rescaled for comparison). Solid, horizontal profiles are threshold velocity, $U_{t}(z, \zeta)$, based on Eq. 3.15, with $\zeta=-0.5$ (black; unstable stratification), $\zeta=0.0 \mathrm{~m}$ (dark grey; neutral stratification), and $\zeta=0.5 \mathrm{~m}$ (light gray; stable stratification).

surface heating further elevates $u_{*}$ and thus $Q$ (Fig. 3). We finally reiterate preceding comments (Sec. 3.1) that these results do not purport to establish unstable conditions as a threshold for mobilization of sediments. Rather, in a mean sense, we have used field data to demonstrate that aeolian saltation is more likely to occur during the day.

\section{Conclusion}

Aeolian saltation is sustained by turbulent winds in the surface layer of the atmospheric boundary layer. Momentum fluxes transported by swirling, eddying motions provide the "gain" needed to mobilize sediment grains such that they are briefly suspended in a ballistic trajectory before impacting on the sediment bed. We have studied a nine month time-series of simultaneous aeolian activity and wind speed measurement from a sandy landscape on the Llano Estacado region. These high-frequency measurements have comprehensively shown that aeolian activity (via saltation for the $\approx 200 \mu \mathrm{m}$ sediment grains that compose sands at the test site) exhibits diurnal variability. We averaged 
the simultaneous wind speed and aeolian activity time-series over each day to attain a representative profile of diurnal variation of both quantities. With this, we used wind speed measurements within the Monin-Obukhov similarity theory to explore how $u_{*}$ at the site would vary during one diurnal cycle. Furthermore, we considered a realistic range of stability parameter, $\zeta$, to demonstrate the role of stratification in setting the ABL $u_{*}$. We demonstrate that the ABL develops its largest $u_{*}$ during the day, when mechanical shear and buoyancy collectively induce turbulent mixing. We cast this result against a candidate value for threshold shear velocity at the site based field data (Stout 2007); this result demonstrates that only during the day - when $\zeta<0$, are conditions favorable for aeolian saltation (i.e., $u_{*}>u_{*, t}$ and $\zeta<0$ ).

The test site (and region) is notorious for strong winds and dust storms. Thus, we also showed how reduction of the streamwise and transverse momentum transport equations can be used to define ABL $u_{*}$ in terms of prevailing regional/mesoscale circulations (embodied by the geostrophic winds, $\boldsymbol{G}$ ). The resulting exercise shows that unstable stratification - by itself - is not necessarily needed to mobilize sediments. Or, initial convective mixing induces the fluctuations needed to elevate $u_{*}$ above $u_{*, t}$, while higher $\boldsymbol{G}$ aloft can provide the additional gains needed to enhance aeolian saltation. That is to say: although aeolian saltation may be occurring throughout the day, $Q$ (Eq. 1.1) will vary significantly throughout the day given its nonlinear dependence on ABL shear velocity, $Q \sim u_{*}^{3}$.

\section{Acknowledgements}

This work was supported by the National Science Foundation (Program Manager: Drs. N. Anderson, C. Lu, and E. Bensman) under Award No. AGS-1500224 (and Summer 2015 REU Supplement Award No. AGS-1542855). Mention of trade names or commercial products in this article is solely for the purpose of providing specific information and does not imply recommendation or endorsement by the U.S. Department of Agriculture. The U.S. Department of Agriculture is an equal opportunity provider and employer.

\section{REFERENCES}

Bagnold, R.A. 1937 The transport of sand by wind. Geographical Journal 89 (5), 409-438. Bagnold, R.A. 1956 The Physics of Blown Sand and Desert Dunes. Chapman and Hall.

Bomar, G.W. 1983 Texas Weather. University of Texas Press.

Brutsaert, W. 2005 Hydrology: an Introduction. Cambridge University Press.

Businger, J.A., WyngaArd, J.C., Izumi, Y. \& Bradley, E.F. 1971 Flux-profile relationships in the atmospheric surface layer. J. Atmos. Sci. 28, 181-189.

Calaf, M., Meneveau, C. \& Meyers, J. 2010 Large eddy simulation study of fully developed wind-turbine array boundary layers. Phys. Fluids 22, 015110.

Csanady, G.T. 1974 Equilibrium theory of the planetary boundary layer with an inversion lid. Boundary Layer Meteorol. 6, 63-79.

Cushman-Roisin, B. \& Beckers, J.-M. 2011 Introduction to Geophysical Fluid Dynamics. Academic Press.

DEARdorfF, J.W. 1972 Numerical investigation of neutral and unstable planetary boundary layers. J. Atmos. Sci. 29, 91-115.

Frandsen, S., Barthelmie, R., Pryor, S., Rathmann, O., Larsen, S., Hojstrup, J. \& Thogersen, M. 2006 Analytical modelling of wind speed deficit in large offshore wind farms. Wind Energy 9.

Frank, A. \& Kocurek, G. 1994 Effects of atmospheric conditions on wind profiles and aeolian sand transport with an example from white sands national monument. Earth Surface Processes and Landforms 19, 735-745. 
GarRatt, J.R. 1994 The atmospheric boundary layer. Cambridge University Press, UK.

HOLLIDAY, V.T. 2001 Stratigraphy and geochronology of upper Quaternary eolian sand on the Southern High Plains of Texas and New Mexico, United States. Bulletin of the Geological Society of America 113, 88-108.

Horst, T.W., Kleissl, J., Lenschow, D.H., Meneveau, C., Moeng, C.H., Parlange, M.B., Sullivan, P.P. \& Weil, J. C. 2004 Hats: Field observations to obtain filtered fields from crosswind arrays of sonic anemometers in the atmospheric surface layer. $J$. Atmos. Sci. 61, 1566-1581.

Kleissl, J., Kumar, V., Meneveau, C. \& Parlange, M.B. $2004 a$ Numerical study of the dynamic smagorinsky models in large-eddy simulation of the atmospheric boundary layer: Validation in stable and unstable conditions. Water Resour. Res. 42, W06D10.

Kleissl, J., Parlange, M.B. \& Meneveau, C. $2004 b$ Field experimental study of dynamic smagorinsky models in the atmospheric surface layer. J. Atmos. Sci. 61, 2296-2307.

Kok, J.F., Parteli, E.J.R., Michaels, T.I. \& Karam, D.B. 2012 The physics of wind-blown sand and dust. Rep. Prog. Phys. 75, 106901.

Kumar, V., Kleissl, J., Meneveau, C. \& Parlange, M.B. 2006 Large-eddy simulation of a diurnal cycle of the atmospheric boundary layer: Atmospheric stability and scaling issues. Water Resour. Res. 42, W06D09.

Lee, J.A., Baddock, M.C., Mbuh, M.J. \& Gill, T.E. 2012 Geomorphic and land cover characteristics of aeolian dust sources in west Texas and eastern New Mexico, USA. Aeolian Res. 3, 459-466.

MAhrT, L. 1998 Stratified atmospheric boundary layers and breakdown of models. Theor. Comput. Fluid Dyn. 11, 263-279.

Martin, Raleigh L., Barchyn, Thomas E., Hugenholtz, Chris H. \& Jerolmack, DouGLAS J. 2013 Timescale dependence of aeolian sand flux observations under atmospheric turbulence. Journal of Geophysical Research: Atmospheres 118, 9078-9092.

Mason, P.J. 1994 Large-eddy simulation: a critical review of the technique. Q. J. R. Meteorol. Soc. 120, 1-26.

Monin, A.S. \& Obuknov, A.M. 1954 Basic laws of turbulent mixing in the ground layer of the atmosphere. Tr. Geofiz. Inst., Akad Nauk SSSR 151, 163-187.

Pahtz, T., KoK, J.F. \& Herrmann, H.J. 2012 The apparent roughness of a sand surface blown by wind from an analytical model of saltation. New Journal of Physics 14, 043035.

Raupach, M.R., Antonia, R.A. \& Rajagopalan, S. 1991 Rough-wall turbulent boundary layers. Appl. Mech. Rev. 44, 1-25.

ShaO, Y. 2008 Physics and Modelling of Wind Erosion. Springer Verlag.

Stout, J.E. 2001 Dust and environment in the Southern High Plains of North America. Journal of Arid Environments 47 (4), 425-441.

Stout, J.E. 2007 Simultaneous observations of the critical aeolian threshold of two surfaces. Geomorphology 85, 3-16.

Stout, J.E. 2010 Diurnal patterns of blowing sand. Earth Surface Processes and Landforms 35, 314-318.

Stout, J.E. \& Zobeck, T.M. 1996 Establishing the threshold condition for soil movement in wind-eroding fields. Proc. Int. Conf. on Air Pollution from Agric. Operations pp. 65-71.

Stull, R.B. 1988 An Introduction to Boundary Layer Meteorology. Kluwer Academic Publishers.

Tennekes, H. \& Lumley, J.L. 1972 A First Course in Turbulence. MIT Press, Cambridge, MA.

WyngaARD, J.C. 1979 The planetary boundary. World Meteorological Organization, Tech. Note 165, Ed. McBean, WMO-No. 530, Geneva, Switzerland p. 201 pp.

WyngaArD, J.C. 2010 Turbulence in the Atmosphere. Cambridge University Press, USA. 